\title{
Dynamically-Tunable Terahertz Band-Stop Filter Based on Multilayer Graphene Metamaterial
}

\author{
Ali Akhavan ${ }^{1}$, Hassan Ghafoori Fard ${ }^{1,2, *}$, Vali Varmazyari ${ }^{1}$ \\ ${ }^{1}$ Department of Electrical Engineering, Amirkabir University of Technology, Tehran, Iran \\ ${ }^{2}$ Photonics Engineering Group, Amirkabir University of Technology, Tehran, Iran
}

\begin{abstract}
In this paper, a dynamically-tunable band-stop filter based on periodically patterned graphene nanostrip and nanodisk in $\mathrm{THz}$ wavelength is proposed and numerically investigated at room temperature. The properties of proposed structure are calculated by using the finite-difference time-domain method. The patterned graphenes are excited by the incident light which leads to absorb two different ranges of spectral wavelength. The simulated results show that a wide free spectral range can be achieved by using multilayer graphene. More importantly, it is found that the transmission dips can be dynamically controlled by adjusting the gate voltage. Moreover, the transmission dips change with graphene mobility which corresponds to graphene intrinsic loss. Finally, the proposed metamaterial structure may be used for many applications such as tunable sensors, active plasmonic switches, and slow light devices.
\end{abstract}

Keywords Graphene Plasmonics, Band Stop Filter, Terahertz Metamaterial, FDTD Method

\section{Introduction}

The Terahertz (THz) range of the electromagnetic spectrum has been explored for a wide variety of applications including biomolecular sensing, wireless communication, spectroscopy and etc. [1-3]. Many natural materials do not react to the $\mathrm{THz}$ radiation, therefore the $\mathrm{THz}$ waves are not most commonly used in comparison with other waves. Whereas, metamaterials, which are artificially made by scientists, can be used in THz range [4]. The optical properties of metamaterial such as periodicity and dielectric properties, let us to control and manage the $\mathrm{THz}$ waves and devices [5, 6]. For these reasons, many types of research have been numerically investigated based on metamaterials at $\mathrm{THz}$ range [7-9]. Designing of $\mathrm{THz}$ devices are one of the common approaches in metamaterials that have active control over the plasmon resonance. It causes that materials were built which have dynamic optical properties such as liquid crystal and nonlinear media [10, 11]. Unfortunately, the refractive index changes of these materials are low by applying mechanical forces and electrical biases. Therefore, the active plasmonic devices that are built with these materials, show low optical performances such as high energy consumption, high plasmonic loss, and low operation speed.

Graphene is composed of a single layer of carbon atoms

* Corresponding author:

ghafoorifard@aut.ac.ir (Hassan Ghafoori Fard)

Published online at http://journal.sapub.org/optics

Copyright $\odot 2017$ Scientific \& Academic Publishing. All Rights Reserved arranged in a two-dimensional hexagonal pattern which has emerged as fascinating material with extraordinary properties such as high electron mobility, flexibility and etc. [12-15]. Plasmonic devices based on graphene guide surface plasmon modes with low loss which can be dynamically tuned by changing the electrostatic doping [16, 17]. These unique properties are a suitable candidate to be used in tunable and compact plasmonic devices. Recently, many devices have been theoretically and experimentally studied such as active plasmonic switching with graphene ribbon arrays [18], Fano metamaterial with tuning of the electrical properties of graphene for switching and sensing applications [19], tunable infrared plasmonic devices with graphene/insulator stacks [20], switching terahertz waves with gate-controlled graphene metamaterials and electromagnetic response [21] and broadband ultrafast dynamics of few-layer epitaxial graphene [22].

In this paper, a tunable band-stop filter with periodic structure consists of nanostrip and nanodisk graphenes. Two patterns of graphene are excited by the incident wave at the same time and absorb two different spectrum wavelengths. The surface conductivity of single-layer graphene is modeled which can be used to simulate the device performance by using the finite difference time-domain (FDTD) method. Then, structure geometry of proposed tunable band-stop filter is presented. After that, important geometric parameters of structure and graphene are considered in order to investigate the filter transmission spectra. Finally, the performance of presented device is investigated in optical telecommunication application, especially in optical switching. 


\section{Graphene Surface Conductivity Model}

In this section, the properties of graphene surface conductivity are introduced. A complex surface conductivity is used for modelling of graphene. The surface conductivity contains intra-band and inter-band transitions.

At the $\mathrm{THz}$ range, it can be calculated with random phase approximation, according to Kubo formula [23]:

$$
\begin{gathered}
\sigma\left(\omega, \mu_{c}, \tau, T\right)=\sigma_{\text {intra }}\left(\omega, \mu_{c}, \tau, T\right)+\sigma_{\text {inter }}\left(\omega, \mu_{c}, \tau, T\right) \\
\sigma\left(\omega, \mu_{c}, \tau, T\right)=\frac{j}{\omega+\frac{j}{\tau}} \frac{e^{2} 2 k_{B} T}{\pi \hbar^{2}} \ln \left[2 \cosh \frac{\mu_{c}}{2 k_{B} T}\right] \\
+\frac{e^{2}}{4 \hbar}\left[G\left(\frac{\hbar \omega}{2}\right)+j \frac{4 \hbar \omega}{\pi} \int_{0}^{\infty} \frac{G(\xi)-G(\hbar \omega / 2)}{(\hbar \omega)^{2}-4 \xi^{2}} d \xi\right] \\
G(\xi)=\frac{\sinh \left(\xi / k_{B} T\right)}{\left[\cosh \left(\mu_{c} / k_{B} T\right)+\cosh \left(\xi / k_{B} T\right)\right]}
\end{gathered}
$$

where $e, \omega, T, \mu_{\mathrm{c}}, \hbar$ and $\tau$ are the electron charge, the radian frequency, temperature, Fermi level energy, plank constant and carrier relaxation time, respectively.

Carrier relaxation time is evaluated by $\tau=\mu \mu_{c} / e v_{F}^{2}$, in which $\mu$ is dc mobility and $\mathrm{v}_{\mathrm{F}}=10^{6} \mathrm{~m} / \mathrm{s}$ (Fermi velocity in graphene) [19]. Also, the reported model can be used for multilayer graphene. In the multilayer graphene, the surface conductivity is achieved by scaling the conductivity in number of layers in equation (1) [20, 24]. Actually, graphene mobility depends on its preparation method. In previous reports, $\mu=230000 \mathrm{~cm}^{2} / V$.s has been experimentally achieved in suspended graphene [25]. High value of graphene mobility on $\mathrm{SiO}_{2}$ substrate has been reported $\mu=40000$ $\mathrm{cm}^{2} / V$.s [26]. In recent researches, the combination of hexagonal boron nitride (h-BN) and graphene have been introduced, which can improve electrical properties of graphene. Because h-BN has a few surface trapped charges which has only $1.8 \%$ lattice mismatch with graphene [27]. In the simulation, the device performance is assumed at room temperature, and $\mu=10000 \mathrm{~cm}^{2} / V$.s.

\section{Structure Geometry and Simulation Method}

Figure 1(a) illustrates the conceptual schematic of designed graphene plasmonic band-stop filter. The geometry of unit cell is depicted in figure 1(b). The patterned graphene is deposited on the substrate of silicon dioxide with relative permittivity 2.09. The unit cell has a square lattice with period array $\mathrm{P}=1 \mu \mathrm{m}$. The radius of graphene nanodisk is $\mathrm{R}=200 \mathrm{~nm}$, also the length and width of graphene strip have been assumed $\mathrm{L}=600$ and $\mathrm{W}=150 \mathrm{~nm}$, respectively. A normally incident y-polarized wave (i.e., the electric field component parallel to y-direction) is used for excitation of surface plasmon modes [28].

The surface plasmon modes propagate along dielectric-graphene interface and form a standing wave along graphene layer. The periodic boundary conditions are applied in $\mathrm{x}$ and $\mathrm{y}$ directions, while a perfect matched layer
(PML) boundary is used in the z-direction. The transmission spectra $T(\omega)=\left|S_{21}(\omega)\right|^{2}$ is obtained from S-parameters, and the field distribution is calculated by frequency-domain field profile monitor in $5 \mathrm{~nm}$ above graphene layers.
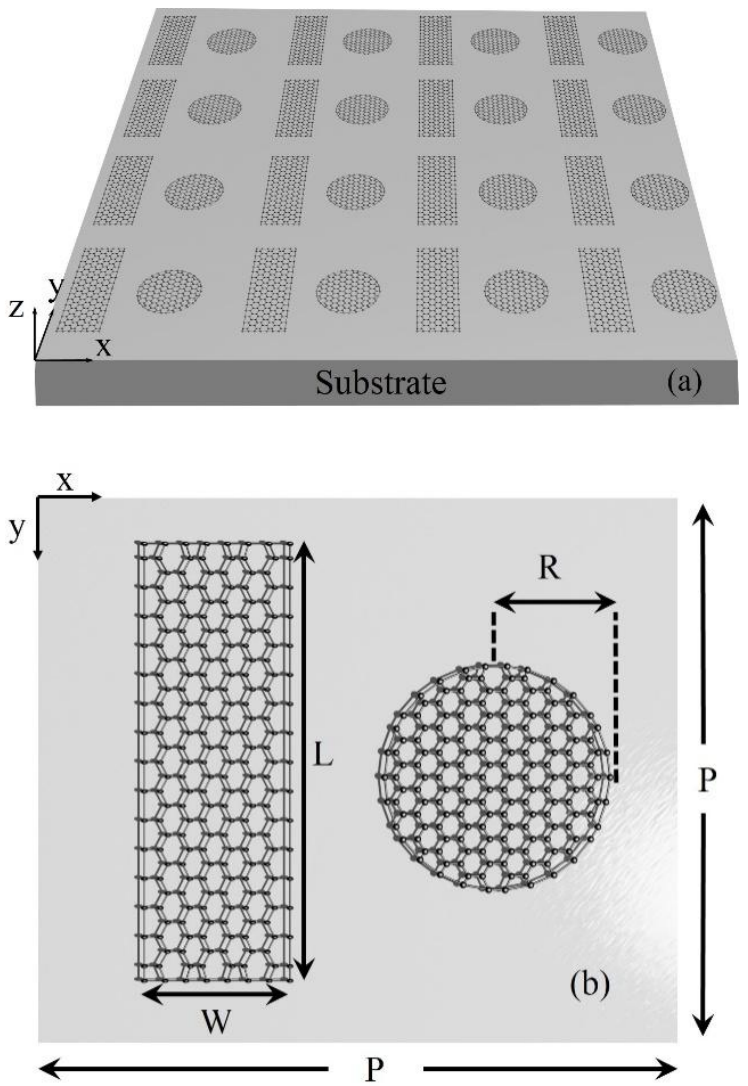

Figure 1. (a) A schematic of periodic graphene nanostructure. (b) A unit cell of the nanostructure. Here the width and length of graphene nanostrip are $\mathrm{W}$ and $\mathrm{L}$, respectively. The radius of graphene nanodisk is $\mathrm{R}$

\section{Results and Discussions}

The transmission spectra of graphene nanostructure is presented in figure 2(a) that shows, the first and second dips are appeared at frequencies of 9.07 and $15.81 \mathrm{THz}$, respectively. Also, figure 2(b) shows the phase of transmission spectra. As demonstrated in this figure, the surface plasmon polariton (SPP) modes are generated in graphene nanostructures. The first and second dips are corresponded to graphene nanostrip and graphene nanodisk, respectively. The z-component of electric field at two dip frequencies are shown in figure 2(c) and 2(d).

In this stage, the effect of structure parameters is considered on transmission spectra. In the first step, the effect of graphene strip length is studied on performance of filter. The resonance frequency of graphene can be interpreted from the quasistatic analysis. The resonance frequency has inversely proportional to graphene length, And expressed as [18]:

$$
f_{r} \propto \sqrt{\frac{\alpha_{0} c \mu_{c}}{2 \pi^{2} \hbar L}} \propto \sqrt{\frac{1}{L}}
$$


where $\alpha_{0}=e^{2} / \hbar c$. Figure 3 demonstrates the transmission spectra with different values of graphene nanostrip length. As can be seen that, by increasing the nanostrip length, the resonance frequency and transmission intensity at the first dip are decreased because the coupling strength is increased.
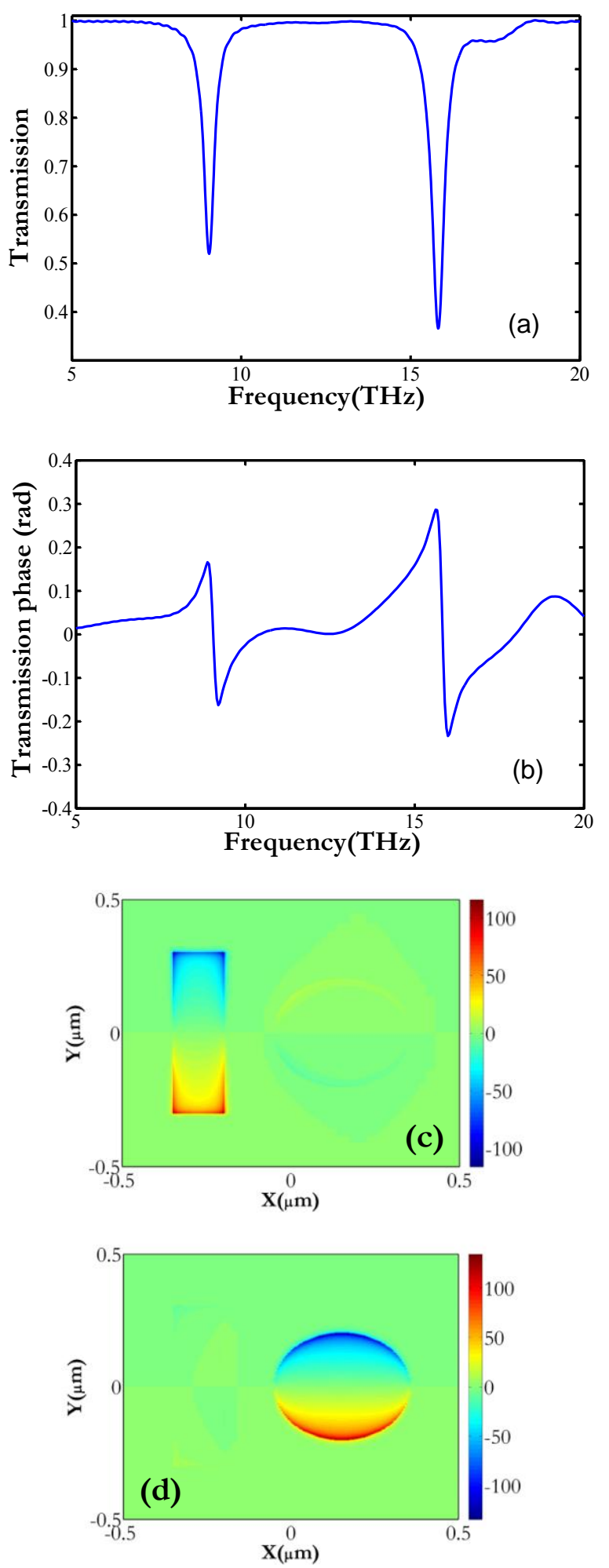

Figure 2. (a) The transmission spectra of the proposed filter. (b) The phase of transmission spectra. (c), (d) z-component of electric field at $9.07 \mathrm{THz}$ and $15.81 \mathrm{THz}$

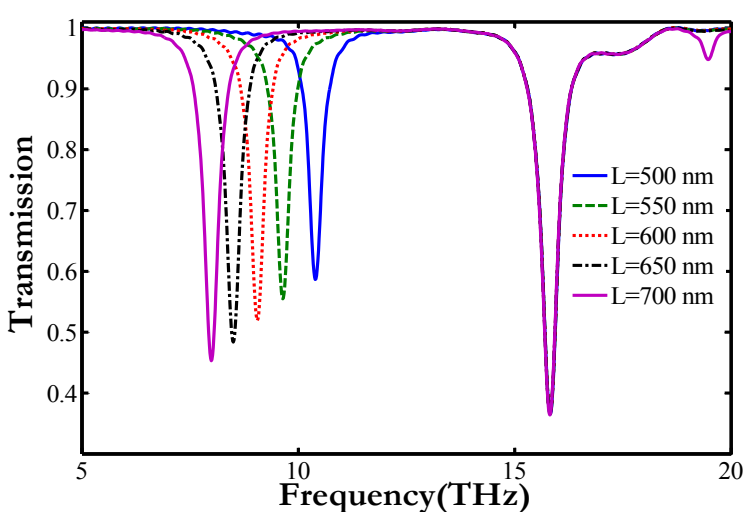

Figure 3. The transmission spectra with different values of graphene nanostrip length $\mathrm{L}$

Also, by applying the changes in nanostrip length, the resonance frequency and transmittance at the second dip remain unchanged.

In the second step, the influence of nanodisk radius is studied on transmission spectra. Figure 4 depicts the transmission spectra for different values of nanodisk radius (R). The resonance frequency and transmission intensity at the second dip are decreased, when the radius of nanodisk increases from 160 to $240 \mathrm{~nm}$. In other word, the second resonance frequency has a red-shift. Unlike the above case, by applying the changes in nanodisk radius, the resonance frequency and transmission intensity at the first dip remain unchanged.

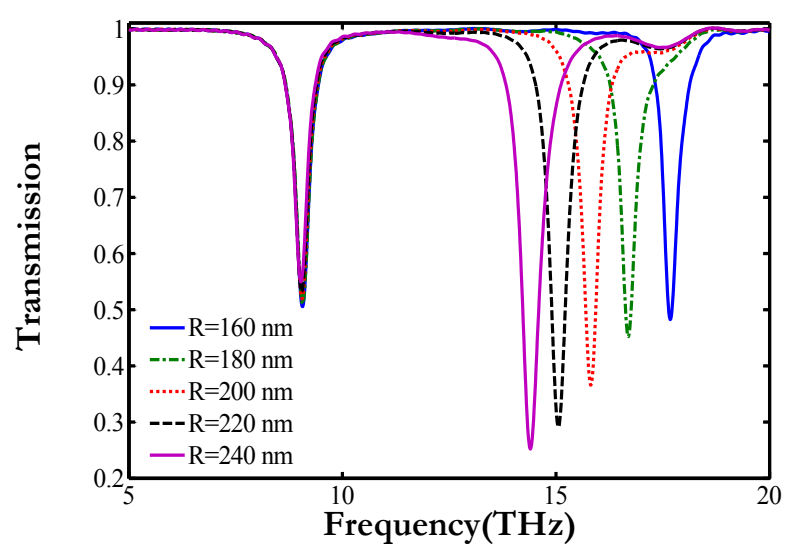

Figure 4. The transmission spectra with different values of graphene nanodisk radius $\mathrm{R}$. The Fermi level energy is $0.6 \mathrm{eV}$

In the third step, the influence of $\varepsilon_{\mathrm{r}}$ is studied on transmission spectrum. Figure 5 shows the transmission spectrum for different values of substrate permittivity from 2 to 4 in step 0.5 . By increasing the permittivity, both resonance frequencies have a red-shift due to increase in total refractive index $\mathrm{n}_{\text {eff }}$. The total shift in the first and second resonance frequencies are $2.69 \mathrm{THz}$ and $4.7 \mathrm{THz}$, respectively.

Finally, the graphene parameters such as Fermi level energy, electron mobility and number of layers are investigated on device performance. The Fermi level energy is considered as the first parameter of them. 


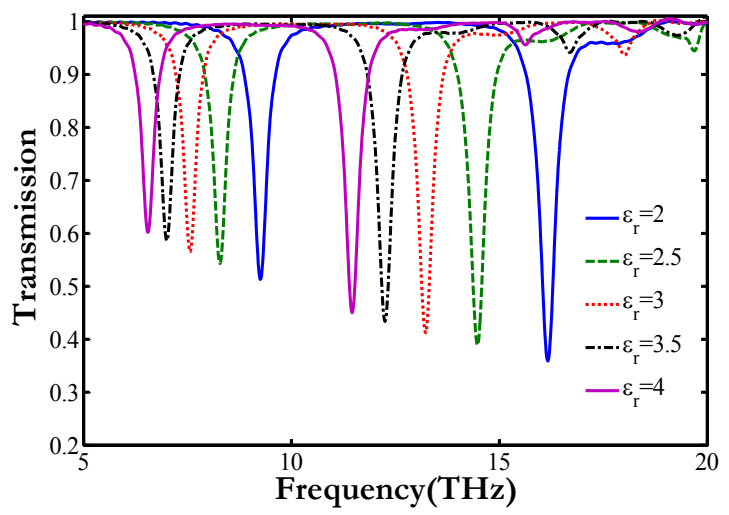

Figure 5. The transmission spectra with different values of substrate permittivity $\varepsilon_{\mathrm{r}}$

According to equation $\mu_{c}=\sqrt{\pi \varepsilon_{r} \varepsilon_{0} V_{a p p} / e t}$, the resonance frequency can be tuned by applied voltage [29]. Where $V_{\text {app }}$ and $t$ are the applied voltage between gating pad and substrate, and thickness of substrate, respectively. Figure 6 shows the obtained transmission spectra of proposed structure under different values of $\mu_{\mathrm{c}}$. It can be clearly seen that the resonance frequencies are gradually blue-shifted with the increase of $\mu_{\mathrm{c}}$. Furthermore, the blue-shift of transmission spectra can be justified by the equation (4).

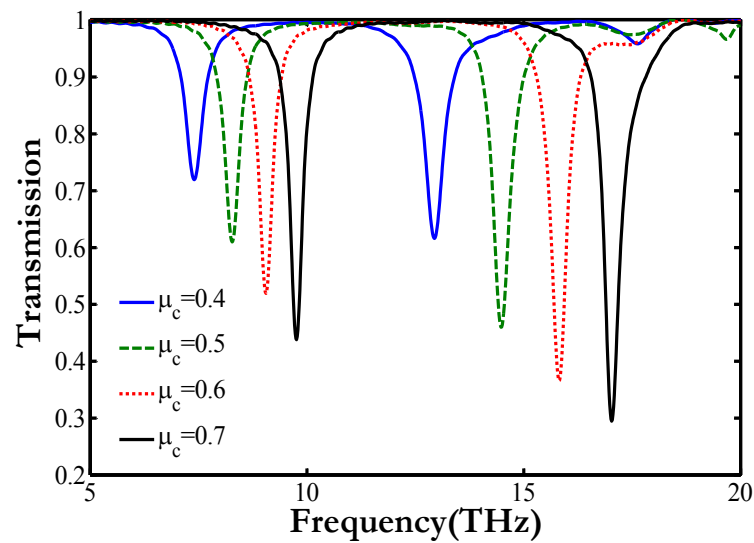

Figure 6. The transmission spectra with different values of graphene Fermi level energy $\mu_{\mathrm{c}}$

In the previous simulated results, graphene has been assumed monolayer. There are some studies by researchers which show that performance of device such as sensitivity is improved by using multilayer graphene [20, 24]. In this subsection, the effect of graphene layers is investigated on device performance. For n-layer graphene, the total conductivity is obtained by $\sigma_{n}(\omega)=n \sigma(\omega)$. Figure 7 shows the transmission spectra for one, two and three layers of graphene. By increasing the number of layers, the resonance frequencies are increased and the transmission dips are became smaller. This property can be used for many applications such as sensors. It can be seen that, the difference between two resonance frequencies are enlarged by increasing the number of layers, it means that, free spectral range (FSR) is increased. FSR in one, two and three layers of graphene are $6.75 \mathrm{THz}, 9.46 \mathrm{THz}$ and $11.46 \mathrm{THz}$, respectively. Finally, the influence of electron mobility $\mu$ is considered on performance of proposed filter which correspond to graphene intrinsic loss. The transmission spectra for four values of mobility is shown in figure 8. For low mobility $\mu$ (high loss), the transmission efficiency is decayed. For actual values of mobility near $10000 \mathrm{~cm}^{2} / V . s$, the efficiency of device is desirable.

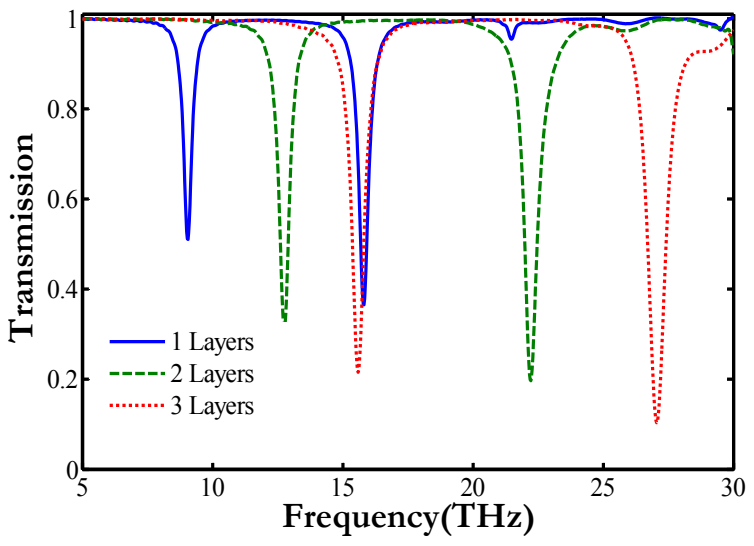

Figure 7. The transmission spectra of $\mathrm{N}$-layer graphene, $\mathrm{N}=1,2,3$

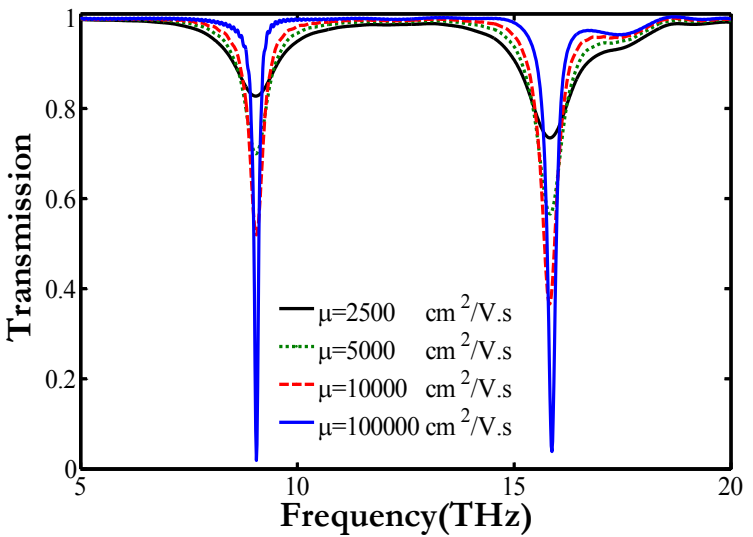

Figure 8. The transmittance of nanostructure for different values of mobility $\mu$
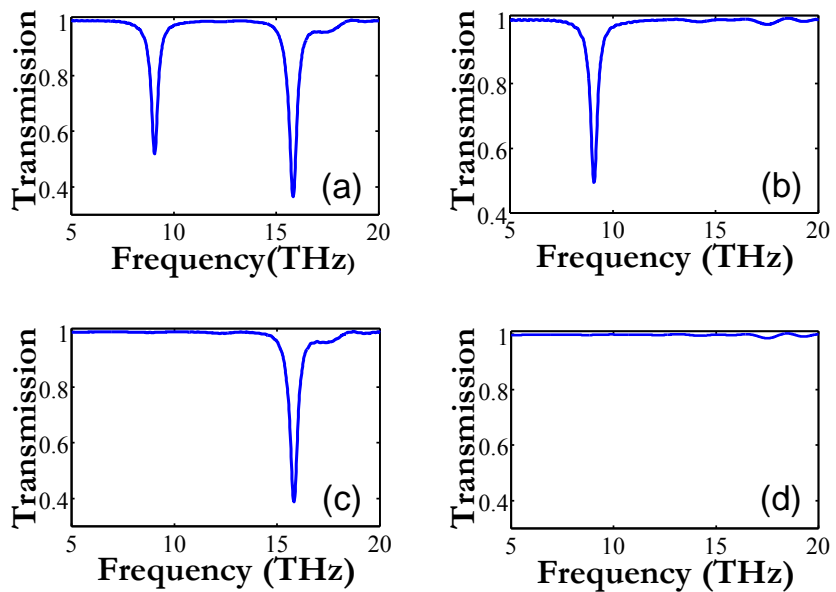

Figure 9. Transmittance of the switch at different states: (a) $\mu_{\mathrm{cl}}=0.6 \mathrm{eV}$ and $\mu_{\mathrm{c} 2}=0.6 \mathrm{eV}$, (b) $\mu_{\mathrm{c} 1}=0.6 \mathrm{eV}$ and $\mu_{\mathrm{c} 2}=0 \mathrm{eV}$, (c) $\mu_{\mathrm{c} 1}=0 \mathrm{eV}$ and $\mu_{\mathrm{c} 2}=0.6 \mathrm{eV}$ and (d) $\mu_{\mathrm{c} 1}=0 \mathrm{eV}$ and $\mu_{\mathrm{c} 2}=0 \mathrm{eV}$ 


\section{Design a Switch Based on Graphene Metamaterial}

In the suggested device, the transmission spectra has been changed by modifying the structure geometry and properties of graphene. Therefore, it can be used for many applications such as optical telecommunication, especially in optical switching. To investigate optical switching performance, the relevant parameters in the presented structure are selected by values of $\mathrm{P}=1 \mu \mathrm{m}, \mathrm{L}=600 \mathrm{~nm}, \mathrm{~W}=150 \mathrm{~nm}, \mathrm{R}=200 \mathrm{~nm}$, $\varepsilon_{r}=2.09$ and $\mu=10000 \mathrm{~cm}^{2} / V$.s. Also, by adjusting the Fermi level energy in two patterns of graphene, the proposed device can be operated as an optical switch at two resonance frequencies of $9.05 \mathrm{THz}$ and $15.81 \mathrm{THz}$.

The values of Fermi level energy are listed in table 1. The Fermi level energy of nanostrip and nanodisk are $\mu_{\mathrm{c} 1}$ and $\mu_{\mathrm{c} 2}$, respectively.

The modulation depth (MD) is used to evaluate the switching performance, which is given as [16]:

$$
M D=\left|\left(T_{o n}-T_{o f f}\right) / T_{o n}\right|
$$

where $T_{o n}$ and $T_{o f f}$ are the magnitude of transmittance at the "on" and "off" states, respectively.

Table 1. Four states of the switch with different Fermi level energy of graphene nanostrip and nanodisk

\begin{tabular}{|c|c|c|c|c|}
\hline States & $\mu_{c 1}(\mathrm{eV})$ & $\mu_{c 2}(\mathrm{eV})$ & $\mathrm{T}_{1}$ & $\mathrm{~T}_{2}$ \\
\hline off-off & 0.6 & 0.6 & 0.52 & 0.37 \\
\hline off-on & 0.6 & 0 & 0.52 & 0.98 \\
\hline on-off & 0 & 0.6 & 0.97 & 0.38 \\
\hline on-on & 0 & 0 & 0.97 & 0.97 \\
\hline
\end{tabular}

The MD for first resonance frequency is 0.48 and second resonance frequency is 0.64 . As can be seen in figure 8 , for increasing $\mathrm{MD}$, the graphene with high mobility (low loss) can be used.

Figures 9(a) - 9(d) show the transmission spectra of the proposed switch at all states. In figure $9(\mathrm{a})$, when $\mu_{\mathrm{cl}}=0.6 \mathrm{eV}$ and $\mu_{\mathrm{c} 2}=0.6 \mathrm{eV}$, two dips are appeared and the switch sets in off state. As shown in Figures 9(b) - 9(c), by setting the appropriate values of $\mu_{\mathrm{c} 1}$ and $\mu_{\mathrm{c} 2}$, the switch is adjusted in "on" state for one of two frequencies. Figure 9(d) shows that the switch is "on" state and all wavelengths are transmitted in which $\mu_{\mathrm{c} 1}=0 \mathrm{eV}$ and $\mu_{\mathrm{c} 2}=0 \mathrm{eV}$.

\section{Conclusions}

In summary, we proposed a dynamically tunable terahertz band-stop filter based on multilayer graphene metamaterial. The graphene metamaterial consists of disk and strip structures. The influences of substrate permittivity and structure parameters on the resonance frequencies and transmission dips were studied in details by FDTD method. The results exhibited that the resonance frequencies can be slightly shifted by adjusting the applied voltage between gating pad and substrate. The effect of graphene layers on the performance of proposed filter was studied. Finally, a switch was proposed for optical telecommunication application, in which the transmission dips are controlled by Fermi level energy. Fortunately, the proposed structure can be fabricated with latest mico-nano fabrication technologies.

\section{REFERENCES}

[1] R. Soref, "Mid-infrared photonics in silicon and germanium," Nat. Photonics, vol. 4, no. 8, pp. 495-497, 2010.

[2] G. P. Williams, "Filling the THz gap — high power sources and applications," Reports Prog. Phys., vol. 69, no. 2, p. 301, 2005.

[3] M. Tonouchi, "Cutting-edge terahertz technology," Nat. Photonics, vol. 1, no. 2, pp. 97-105, 2007.

[4] H.-T. Chen, W. J. Padilla, J. M. O. Zide, A. C. Gossard, A. J. Taylor, and R. D. Averitt, "Active terahertz metamaterial devices," Nature, vol. 444, no. 7119, pp. 597-600, 2006.

[5] H. Tao, W. J. Padilla, X. Zhang, and R. D. Averitt, "Recent progress in electromagnetic metamaterial devices for terahertz applications," IEEE J. Sel. Top. Quantum Electron., vol. 17 , no. 1, pp. 92-101, 2011.

[6] W. Withayachumnankul and D. Abbott, "Metamaterials in the terahertz regime," IEEE Photonics J., vol. 1, no. 2, pp. 99-118, 2009.

[7] J. Gu, R. Singh, X. Liu, X. Zhang, Y. Ma, S. Zhang, S. A. Maier, Z. Tian, A. K. Azad, H.-T. Chen, and others, "Active control of electromagnetically induced transparency analogue in terahertz metamaterials," Nat. Commun., vol. 3, p. 1151, 2012.

[8] N. Born, I. Al-Naib, C. Jansen, R. Singh, J. V Moloney, M. Scheller, and M. Koch, "Terahertz metamaterials with ultrahigh angular sensitivity," Adv. Opt. Mater., vol. 3, no. 5, pp. 642-645, 2015.

[9] R. Singh, I. Al-Naib, D. R. Chowdhury, L. Cong, C. Rockstuhl, and W. Zhang, "Probing the transition from an uncoupled to a strong near-field coupled regime between bright and dark mode resonators in metasurfaces," Appl. Phys. Lett., vol. 105, no. 8, p. 81108, 2014.

[10] A. A. Zharov, I. V Shadrivov, and Y. S. Kivshar, "Nonlinear properties of left-handed metamaterials," Phys. Rev. Lett., vol. 91, no. 3, p. 37401, 2003.

[11] S. Khatua, W.-S. Chang, P. Swanglap, J. Olson, and S. Link, "Active modulation of nanorod plasmons," Nano Lett., vol. 11, no. 9, pp. 3797-3802, 2011.

[12] A. N. Grigorenko, M. Polini, and K. S. Novoselov, "Graphene plasmonics," Nat. Photonics, vol. 6, no. 11, pp. 749-758, 2012.

[13] K. S. Novoselov, V. I. Fal, L. Colombo, P. R. Gellert, M. G. Schwab, K. Kim, and others, "A roadmap for graphene," Nature, vol. 490, no. 7419, pp. 192-200, 2012.

[14] J. Christensen, A. Manjavacas, S. Thongrattanasiri, F. H. L. Koppens, and F. J. de Abajo, "Graphene plasmon 
waveguiding and hybridization in individual and paired nanoribbons," ACS Nano, vol. 6, no. 1, pp. 431-440, 2011.

[15] A. K. Geim and K. S. Novoselov, "The rise of graphene," Nat. Mater., vol. 6, no. 3, pp. 183-191, 2007.

[16] H. S. Chu and C. How Gan, "Active plasmonic switching at mid-infrared wavelengths with graphene ribbon arrays," Appl. Phys. Lett., vol. 102, no. 23, pp. 1-8, 2013.

[17] C. H. Gan, "Analysis of surface plasmon excitation at terahertz frequencies with highly doped graphene sheets via attenuated total reflection," Appl. Phys. Lett., vol. 101, no. 11, p. $111609,2012$.

[18] H.-S. Chu and C. H. Gan, "Active plasmonic switching at mid-infrared wavelengths with graphene ribbon arrays," Appl. Phys. Lett., vol. 102, no. 23, p. 231107, 2013.

[19] M. Amin, M. Farhat, and H. Bağc1, "A dynamically reconfigurable Fano metamaterial through graphene tuning for switching and sensing applications," Sci. Rep., vol. 3, pp. $1-8,2013$.

[20] H. Yan, X. Li, B. Chandra, G. Tulevski, Y. Wu, M. Freitag, W. Zhu, P. Avouris, and F. Xia, "Tunable infrared plasmonic devices using graphene/insulator stacks," Nat. Nanotechnol., vol. 7, no. 5, pp. 330-334, 2012.

[21] S. H. Lee, M. Choi, T. Kim, S. Lee, M. Liu, X. Yin, H. K. Choi, S. S. Lee, C. Choi, S. Choi, X. Zhang, and B. Min, "Switching terahertz waves with gate-controlled active graphene metamaterials," Nat. Mater., vol. 11, no. 10, pp. 1-6, 2012.

[22] H. Choi, F. Borondics, D. A. Siegel, S. Y. Zhou, M. C. Martin, and A. Lanzara, "Broadband electromagnetic response and ultrafast dynamics of few-layer epitaxial graphene," vol. 172102, no. 2009, pp. 1-4, 2012.
[23] V. P. Gusynin, S. G. Sharapov, and J. P. Carbotte, "Magneto-optical conductivity in graphene," J. Phys. Condens. Matter, vol. 19, no. 2, p. 26222, 2007.

[24] C. Casiraghi, A. Hartschuh, E. Lidorikis, H. Qian, H. Harutyunyan, T. Gokus, K. S. Novoselov, and A. C. Ferrari, "Rayleigh imaging of graphene and graphene layers," Nano Lett., vol. 7, no. 9, pp. 2711-2717, 2007.

[25] K. I. Bolotin, K. J. Sikes, Z. Jiang, M. Klima, G. Fudenberg, J. Hone, P. Kim, and H. L. Stormer, "Ultrahigh electron mobility in suspended graphene," Solid State Commun., vol. 146, no. 9, pp. 351-355, 2008.

[26] J.-H. Chen, C. Jang, S. Xiao, M. Ishigami, and M. S. Fuhrer, "Intrinsic and extrinsic performance limits of graphene devices on $\mathrm{SiO}_{2}$," Nat. Nanotechnol., vol. 3, no. 4, pp. 206-209, 2008.

[27] M. Polini and F. H. L. Koppens, "Graphene: Plasmons in moire superlattices," Nat. Mater., vol. 14, no. 12, pp. 1187-1188, 2015.

[28] A. Y. Nikitin, F. Guinea, F. J. Garcia-Vidal, and L. Martin-Moreno, "Surface plasmon enhanced absorption and suppressed transmission in periodic arrays of graphene ribbons," Phys. Rev. B - Condens. Matter Mater. Phys., vol. 85, no. 8, pp. 1-4, 2012.

[29] J. S. Gómez-D’liaz and J. Perruisseau-Carrier, "Graphene-based plasmonic switches at near infrared frequencies," Opt. Express, vol. 21, no. 13, p. 15490, Jun. 2013. 